\title{
Characterization of VOCs and additives in Italian PET bottles and studies on potential functional aldehydes scavengers
}

\author{
Sandro Dattilo ${ }^{1}$ (D) Chiara Gugliuzzo ${ }^{1}$ - Emanuele F. Mirabella ${ }^{1}$. Concetto Puglisi ${ }^{1}$. Andrea A. Scamporrino ${ }^{1}$. \\ Daniela C. Zampino ${ }^{1} \cdot$ Filippo Samperi $^{1}$
}

Received: 14 October 2021 / Revised: 20 January 2022 / Accepted: 22 January 2022 / Published online: 15 February 2022

(c) The Author(s) 2022

\begin{abstract}
This study focused on characterization of Volatile Organic Compounds (VOCs) as contaminants and non-volatile additives in Italian PET bottles, also suggesting potential functional aldehydes scavengers. Several VOCs, such as acetic aldehyde (AA), butanal, 3-methyl butanal, 1,3-dioxolane, pentanal, hexanal, octanal, 5-hepten-2-one, nonanal, and decanal, were identified by Head Space-Gas Chromatography/Mass Spectrometry (HS-GC/MS) in the PET bottles used for the packaging of six Italian brands mineral waters. AA, 1,3-dioxolane, octanal, 5-hepten-2-one, nonanal, and decanal were the most abundant compounds identified. These contaminants were also identified in the PET-bottled mineral waters. Different experiments using bottle-grade PET pellets (Btlg-PET) and PET bottles' fragments with and without the addition of epoxidized soybean oil (ESBO) or erucamide as lubricant/plasticizer additives, poly (m-xylene adipamide) (MXD6), and/or anthranilamide (2-aminobenzamide) as potential aldehydes scavengers were carried out. Mostly VOCs observed in the PET bottles analysed were identified in a neat ESBO sample. The presence of the ESBO additive in the PET-bottle fragments was also observed by matrix-assisted laser desorption/ionization time of flight mass spectrometry analysis (MALDI-TOF MS). The ESBO sub-products were not observed in the virgin btlg-PET pellets analysed by both HS-GC/MS and MALDI-TOF MS. These results suggest that the VOCs come from an ESBO additive probably loaded during the blow-moulding processes used for the manufacturing of PET bottles. Further studies established that MXD6 (1\%w), an efficient oxygen scavenger, could be also used as AA scavenger even in the presence of the commonly used anthranilamide.
\end{abstract}

Keywords PET bottles $\cdot$ VOCs $\cdot$ Mineral waters $\cdot$ ESBO $\cdot$ MXD6 $\cdot$ Erucamide $\cdot$ Anthranilamide $\cdot$ HS-GC/MS

\section{Introduction}

Over the past decades, the worldwide consumption of bottled mineral water has been steadily increasing due to a growing demand of consumers to drink safe water having controlled chemical and microbiological quality [1,2]. Different types of packaging materials have been developed; nevertheless, plastic packaging has been preferred over glass in almost all countries. In particular, the poly(ethylene terephthalate) (PET) is widely used in beverage-bottling applications [1] due to its flexibility, lightweight, clarity, excellent tensile and

Sandro Dattilo

sandro.dattilo@cnr.it

$\triangle$ Filippo Samperi

filippo.samperi@cnr.it

1 Institute for Polymers Composites and Biomaterials, IPCB CNR, Via Paolo Gaifami, 18, 95126 Catania, Italy impact strength, chemical and thermal resistance, negligible permeability to carbon dioxide [3, 4], ease of processing and coloration, and low cost $[1,2,5-7]$.

The bottle-grade PET (btlg-PET) is produced by a catalysed polycondensation reaction of dimethyl terephthalate (DMT) or terephthalic acid (TPA) with ethylene glycol (EG) at about $280{ }^{\circ} \mathrm{C}$ in the presence of some co-monomers such as diethylene glycol (DEG) and isophthalic acid (IPA). These co-monomers are widely used to minimize polymer thermal crystallization during the production of pre-forms and the blow-molding process used to obtain the PET bottles $[7,8]$. Indeed, they reduce the size of spherulites and, as a result, the final container, such as the PET bottles analysed, is transparent. The concentration of DEG units in the btlg-PET changes from 1 to $4 \mathrm{~mol} \%[7,8]$ as a function of the polymerization conditions. These units can also derive from the etherification reaction between two EG molecules with elimination of water during the polymerization process. 
Their concentration influences the chemical and barrier properties of the PET. It is known that the DEG units are reactive along the chains of PET, allowing the thermal and thermo-oxidative degradation of PET [8-17], and are the precursor to colour formation in btlg-PET [8].

PET is sensitive to heat and oxidation that take place during its manufacturing processes due to different types of chemical reactions that can occur [8-17]. In particular, high temperature and presence of oxygen can generate thermomechanical and thermo-oxidative reactions, whereas trace of water can induce hydrolysis during the melt process [15]. The different degradation mechanisms can generate sub-products that could remain trapped inside the polymer matrix. Under the conditions of temperature and pressure used for polymer transformation into a final food-container, these substances could be released to beverage/food they package.

To ensure the safety of the plastic materials used in contact with foodstuffs must be provided the declaration of conformity according to the European regulation No. 10/2011 [18]. This regulation lists the substances authorized for use in plastic materials and establishes the compounds subject to restrictions and/or specifications according to their toxicological action.

Several chemical substances have been identified in the PET-bottled mineral waters which came from the PET-bottle, as terephthalic acid and ethylene glycol (raw materials), antimony (polymerization catalyst), oligomers (polymerization products, mainly trimers and up to decamers), and acetic aldehyde (AA) [19-41]. The formation of AA occurs during the high-temperature processing of PET for the production of bottles due to the degradation reactions that involve the EG units either along the chains or as terminals [10-14]. Among the volatile products, AA is the most dominant one and has a distinct odour and taste, generally described as sweet plastic-like, with a low sensory detection threshold [27]. It is a potential human carcinogen, and it is very important to check its amount in freshly blown PET before use in the bottling drink industry [21-24, 42].

The analysis of PET reveals some volatile organic contaminants (VOCs), produced by authorized initial reactants and additives [21, 25, 32, 33, 37]. Genotoxic and estrogenic activities in PET-bottled water have been reported [21, 24, $32,33]$. Chemical mixtures in bottled water have been suggested as the source of these toxicological effects. Nevertheless, the migration of monomers, additives, and their degradation products from plastic materials can never be totally prevented. PET is considered highly inert compared to other plastics used for the packaging of food and beverages [1, 2,5]; however, several studies have shown the presence of unexpected substances in PET-bottled water such as shortand medium-chain aldehydes [19, 25, 28, 36, 37], ketones, aliphatic and aromatic hydrocarbon, and terpenes [19]. The origin of these compounds has not been clearly recognized and it has been hypothesized that they may come from capsealing resins, PET container, recycled PET, background contamination, water processing steps, VOCs, etc. [19, 25, $28,29,36,37,40]$. Many studies have been carried out to identify the compounds which migrate from the PET bottle to the beverages and, in particular, to the mineral water [19-44]. Volatile aldehydes (formaldehyde and AA) and antimony are clearly related to migration from PET bottles into water. The studies have been mainly focused on the determination of short-chain carbonyl compounds, such as formaldehyde, AA, and the phthalates due to their carcinogenic and estrogenic effects [21, 24, 42]. Cincotta et al. [19] developed an analytical method based on headspace solidphase microextraction-gas chromatography-mass spectrometry (HS-SPME-GC-MS) to characterize the NIAS present in some commercial Italian mineral water packaged in PET bottles. The volatile organic compounds were identified and quantified in water samples at different storage times at room and stressed temperature. Saturated and 2-unsaturated aliphatic aldehydes, ketones, terpenes, phthalates, aliphatic, and aromatic hydrocarbons were identified and determined, most of them for the first time, in PET-bottled mineral water [19]. The concentrations of the NIAS recognized showed a significant increase during the water-bottled shelf-life. In particular, the most abundant compounds identified were decanal and nonanal. Based on the identified substances, the authors hypothesized their migration not only from packaging but also from the closure caps and adhesive. Moreover, the recycling of PET bottles has been greatly developed over the last years [2, 30, 43, 45]. Most of recycled PET materials (r-PET) have been used in packaging application ( $26 \%$ in bottles, $11 \%$ in strapping, and $38 \%$ in trays), whereas the remaining $25 \%$ for fleece fill [5]. Recently, van Velzen et al. [43] have determined the migration to mineral water of several substances from PET bottles produced using three types of r-PET. Beside the well-known migrants (AA, EG, 2-methyl-1,3-dioxolane), they also detected the migration of limonene, acetone, butanone, furan, benzene, and styrene [43]. The presence of these contaminants was attributed both to the composition of the recycled PET materials and to the industrial process to produce the bottles. The formation of benzene in the r-PET was attributed to polyvinylchloride as contaminant.

Considering the literature results, in the present work, we investigated not only the VOCs present in the PET bottles used for the packaging of some commercial Italian mineral water but also the volatile compounds present in the raw bottle-grade PET (btlg-PET) pellets. To identify eventual potential lubricants used for the production of PET bottles, we melt-mixed btlg-PET pellets with different amounts of epoxidized soybean oil (ESBO) (1\%) and erucamide (1\%w). Moreover, blends of btlg-PET loaded with low amount 
( $1 \% \mathrm{w})$ of poly (m-xylene adipamide) (MXD6) and/or anthranilamide (2-aminobenzamide) were prepared to investigate their potential AA scavenger effect and to identify eventual VOCs they could generate.

\section{Materials and methods}

\section{Samples and chemicals}

The PET bottles used for the packaging of six different Italian brands mineral waters were purchased from several local stores. The different water bottles, which did not report information about their manufacturing conditions and/or presence of r-PET, are referred as PET-btl-n (with $\mathrm{n}$ from 1 up to 6) in the text. The btlg-PET pellets, the anthranilamide and the erucamide were purchased by Merch-Sigma Aldrich (Italy). MXD6 S6007 commercial grade (M.F.R. $2.0 \mathrm{~g} / 10$ min-ASTM D1238 method) was supplied by Mistubishi Gas Chemical with $\mathrm{S} 6007$ commercial grade (M.F.R. $2.0 \mathrm{~g} / 10$ min-ASTM D1238 method). The ESBO sample was gently donated by Proplast-Plastic Innovation Pole (Alessandria, Italy). MALDI matrix (trans-2-[3-(4-tertButylphenyl)-2-methyl-2-propenylidene]malononitrile, referred as DCTB), both deuterated chloroform $\left(\mathrm{CDCl}_{3}\right)$ and trifluoroacetic acid (TFA-d), tetrachloroethane (TCE), and tetramethylsilane (TMS) were purchased by Merch-SigmaAldrich (Italy).

\section{Melt-mixing process}

The processing in the molten state $\left(280{ }^{\circ} \mathrm{C}\right)$ of all samples was carried out by a Brabender mixer AEV 3301983 at $35 \mathrm{rpm}$ for different times $(5,10$, and $20 \mathrm{~min})$ under inert argon flow to avoid the thermo-oxidative processes. Typically, $40 \mathrm{~g}$ of PET samples were melt-mixed with and without specific additives. About $5.0 \mathrm{~g}$ of blends were sampled from the Brabender cup at 5, 10, and 20 min and analyzed by HS-GC/MS without pre-treatment. Some melt-mixed samples were also analyzed by MALDI-TOF MS.

\section{Selective extraction}

The neat PET-bottle fragments and the btlg-PET/MXD6 (1\%w) blends melt-mixed for $5 \mathrm{~min}$ at $280^{\circ} \mathrm{C}$ were selectively extracted with TCE at $60^{\circ} \mathrm{C}$ for $6 \mathrm{~h}$. In particular, $0.5 \mathrm{~g}$ of each sample was treated with $20 \mathrm{~mL}$ of TCE, and then filtered and washed several times with the same fresh solvent. The TCE solutions were rotoevaporated and the solid materials recovered were analyzed by MALDI-TOF MS.

\section{Head space gas chromatography/mass spectrometry (HS-GC/MS)}

HS-GC/MS analysis was performed by a Turbomatrix HS40 Trap (Perkin Elmer, Wellesley, MA). In this technique, the headspace sampling by a trap is connected to a GC-2020 system (Shimadzu Corporation), coupled with a triple quadrupole mass spectrometry detector TQ8040 (Shimadzu Corporation) with electronic ionization $(70 \mathrm{eV})$. The gas chromatograph was equipped with a Capillary Column (Zebron ZB-WAX Phenomenex, stationary phase $100 \%$ Polyethylene Glycol, inner diameter of $250 \mu \mathrm{m}$, film thickness of $0.25 \mu \mathrm{m}$, and a length of $30 \mathrm{~m}$ ). The carrier gas was helium at a controlled flow of $1.78 \mathrm{~mL} / \mathrm{min}$. The split ratio was $1 / 50$ of the total flux. The HS-GC/MS analyses of the bottle fragments (bottle clippings of roughly $0.5 \times 0.5 \mathrm{~cm}$ ) were carried out without grinding to avoid eventual cross-contamination in the mill with substances from previous samples as well as uncontrolled loss of volatile substances like AA. $1.0 \mathrm{~g}$ of each bottle fragments, btlg-PET pellets, and melt-mixed blends was introduced in a glass vial, and crimped and equilibrated at $100{ }^{\circ} \mathrm{C}$ for $15 \mathrm{~min}$ in a HS oven system. After equilibration, the headspace of the vial was injected on the gas chromatograph. The following HS sampler conditions were applied: oven temperature $100^{\circ} \mathrm{C}$, loop: $100^{\circ} \mathrm{C}$, transfer line $110^{\circ} \mathrm{C}$, vial equilibration $15 \mathrm{~min}$, MS ion source temperature $200^{\circ} \mathrm{C}$ and interface $250^{\circ} \mathrm{C}$. The GC oven conditions were the following: temperature at $30^{\circ} \mathrm{C}$ for $5 \mathrm{~min}$, ramp to $120^{\circ} \mathrm{C}$ at $6{ }^{\circ} \mathrm{C} / \mathrm{min}$, isotherm and stabilization at $120^{\circ} \mathrm{C}$ for $10 \mathrm{~min} .10 \mathrm{~mL}$ of each different packaged mineral water were placed into a $22 \mathrm{~mL}$ sealed vial and analyzed as described above, without pre-treatment. The blank measurement before each analysis was carried out with the same method using an empty vial in the oven of HS. Standards and mixture of them were analyzed in the same experimental conditions. Each sample was analyzed almost in triplicate.

\section{Matrix-assisted laser desorption/ionization time of flight mass spectrometry (MALDI-TOF MS)}

The MALDI-TOF MS analyses were performed in reflectron mode by a 4800 Proteomic Analyzer (Applied Biosystems) MALDI-TOF/TOF instrument equipped with a Nd:YAG laser at a wavelength of $355 \mathrm{~nm}$ with $<500 \mathrm{ps}$ pulse and $200 \mathrm{~Hz}$ firing rate. The accelerating voltage was $15 \mathrm{kV}$. External calibration was performed using an Applied Biosystems calibration mixture consisting of polypeptides with different molecular weight values. The irradiance was maintained slightly above the threshold to obtain a mass resolution of about $6000-8000$ fwhm. Mass accuracy was about $30-50 \mathrm{ppm}$ in the mass range from $\mathrm{m} / \mathrm{z} 50$ up to 1500 . The DCTB matrix was used to analyse the extracted PET 
samples. Mass spectra were elaborated by the Data Explorer software (Applied Biosystems).

\section{${ }^{1}$ H-NMR}

${ }^{1} \mathrm{H}$-NMR spectra of the PET-bottle fragments were recorded by a Bruker Avance 400 spectrometer. The analyses were performed at $25^{\circ} \mathrm{C}$ at $400.13 \mathrm{MHz}$. Samples were solved in a deuterated $\mathrm{CDCl}_{3} / \mathrm{TFA}-\mathrm{d}$ mixture $(75 / 25 \mathrm{v} / \mathrm{v})$ at the concentration of $10 \mathrm{mg} / \mathrm{ml}$. The TMS was used as internal standard.

\section{Results and discussion}

\section{Identification of VOCs from bottled Italian waters and PET bottles}

The aim of this study was focused on the investigation and the determination of VOCs present not only in bottled mineral waters but, in particular, in the PET bottles used for the packaging of six commercial mineral waters and in the raw bottle-grade PET. Previous studies highlighted the presence of VOCs in commercial mineral waters [6, 19, 21, 25-29, 32-34, 36-38]. It was supposed that some volatile organic compounds identified in the packaged mineral waters may come from the PET bottles and mainly from the caps and the adhesive used for sticking the bottle labels $[3,4,6,19$, $25,27,36,37,40]$. To verify this supposition and check whether VOCs could arise from some additives used during the manufacturing of the PET bottles studied, we analyzed six commercial Italian brand mineral waters packaged in PET bottles and the corresponding PET bottles in which the waters were therein. Different bottles $(1.5-2.0 \mathrm{~L})$ of nocarbonated mineral were analyzed. The volatile compounds trapped in the packaging material (PET bottles) were analyzed using bottle fragments distant from the closure caps and the adhesives (Fig. S1). Each volatile compound was identified using a mass spectral database (i.e., library mass spectra database: NIST 14 and Wiley database) with a match higher than $90 \%$ and some of them also by injection of its analytical standard (Fig. S2).

All the mineral water brands analyzed, stored either at room temperature (RT, $25^{\circ} \mathrm{C}$ ) and at $60^{\circ} \mathrm{C}$ for $1 \mathrm{~h}$ up to $45 \mathrm{~h}$, showed very similar chromatograms. Figure 1 reports the chromatograms from the mineral water packaged in the PET-btl-1, kept for $45 \mathrm{~h}$ either at RT (Fig. 1a) and $60{ }^{\circ} \mathrm{C}$ (Fig. 1b), and from the fragments of PET-btl-1 (Fig. 1c) in which the water was therein. The chromatogram of the bottled mineral water stored at RT (Fig. 1a) shows traces of AA (peak 1), butanal (peak 3), 3-methyl butanal (peak 4), 1,3-dioxolane (peak 5), and pentanal (peak 6). The intensity of these volatile compounds increases in the bottled water stored at $60{ }^{\circ} \mathrm{C}$ (Fig. 1b), according to literature data. The compounds 9-15 (hexanal, octanal, 5-epten-2-one-6, 6-methyl, nonanal, and decanal) observed at higher retention times (from 4 up to $25 \mathrm{~min}$ ) in the chromatogram of the bottled mineral water stored at $60{ }^{\circ} \mathrm{C}$ were not present in that relative to RT stored water. Indeed, high temperature enhances the process of migration of volatile carbonyl compounds (i.e., AA, saturated aldehydes, and ketones) from PET bottles into mineral water, as previously reported $[19,25,26,29,31,32,36,37]$. At high retention time was also found the presence of ethylene glycol (EG). Besides the expected AA, other VOCs, such as aliphatic aldehydes (pentanal, hexanal, octanal, nonanal, and decanal), ketone (6 methyl-5-hepten-2-one) and 1,3-dioxolane were detected in the fragments of PET-btl-1 (Fig. 1c). Long-chain aldehydes, such as nonanal and decanal, were the most abundant compounds identified, as previously observed in Italian bottled waters [19].

The HS-GC/MS chromatograms of the bottle fragments from the PET bottles (namely PET-btl-1, PET-btl-2, PETbtl-3, PET-btl-4, PET-btl-5, and PET-btl-6) in which the six Italian brands of mineral water were therein are showed in Fig. S3. Table 1 shows all the VOCs identified, which correspond to the labeled peaks in Figs. 1 and S3, with the respective retention times and the normalized relative intensity.

\section{Determination of additives in PET bottles}

The analyses of the PET-bottled mineral waters analysed (Fig. 1) highlighted the presence of VOCs such as AA(1), acetone (2), butanal (3), 3-methyl butanal (4), 1,3-dioxolane (5), pentanal (6), hexanal (9), octanal (12), 5-hepten-2-one, 6-methyl (13), nonanal (14), and decanal (15). Since these organic contaminants were found in all PET bottles, we believe that these substances come from the PET bottles exclusively. VOCs' provenience from additives present in the PET bottles was analyzed by additional studies.

First, the bottle fragments were selectively extracted using TCE as solvent and the soluble compounds were analyzed by MALDI-TOF MS. All soluble samples extracted from the different PET bottles showed very similar mass spectra. Table S1 lists the main PET oligomers identified by MALDI-TOF MS in the extracted PET-bottle fragments, whereas Fig. 2 reports the MALDI-TOF mass spectrum of the soluble components extracted from the PET-btl-1, as an example. The sodiated ions of the oligomeric species found are labeled with different symbols. Figure 2 shows intense mass peaks belonging to cyclic PET oligomers (species An) which are detected as sodiated and protonated macromolecules (Table S1). Cyclic oligomers having one diethylene glycol (DEG) unit in the backbone (species A'n) and low abundant sodiated ions from the linear PET oligomers were also detected. Moreover, species Bn, having hydroxyl 


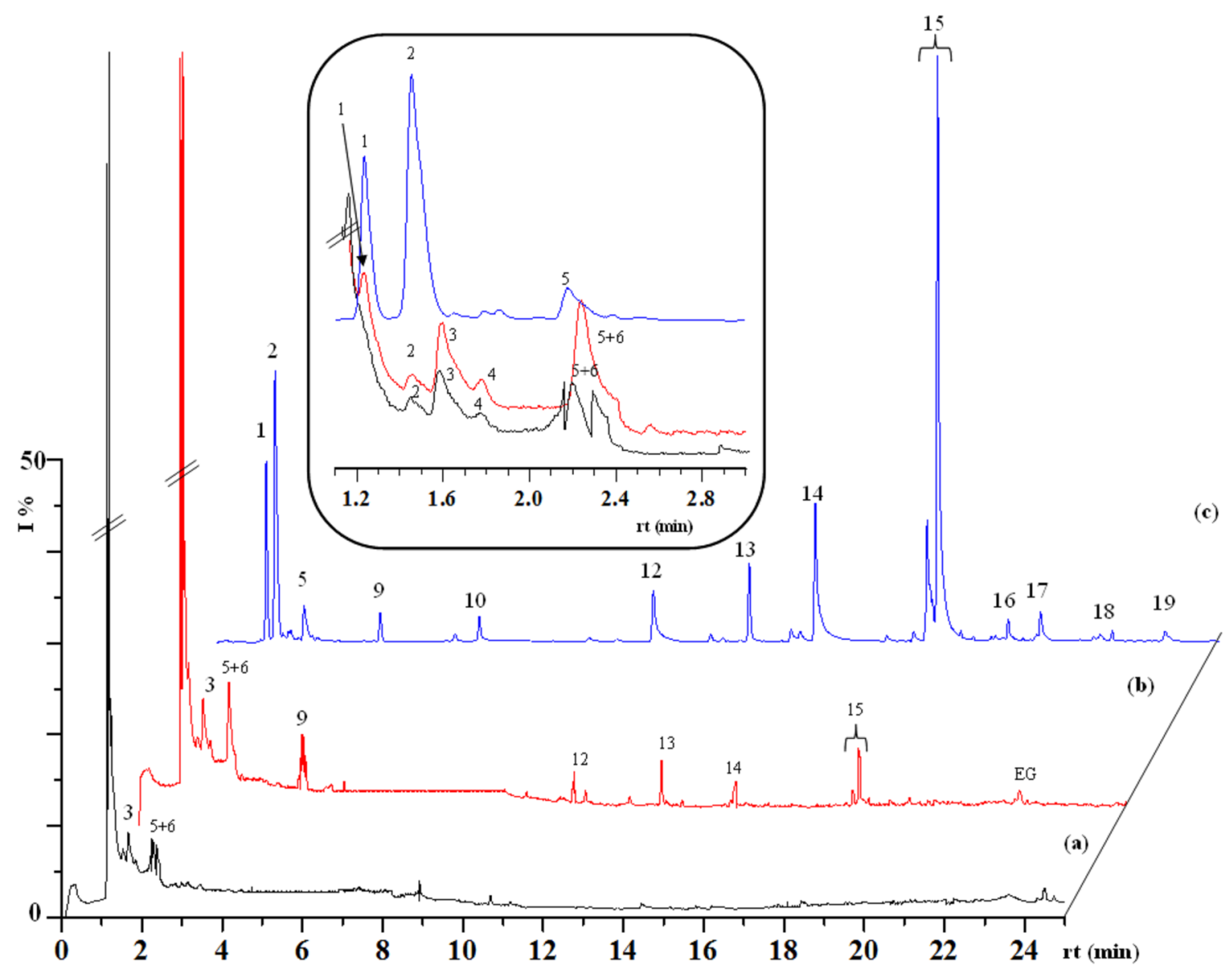

Fig. 1 Gas chromatograms of HS-GC/MS analysis of a mineral water packaged in the PET-btl-1 stored both at a RT and b $60{ }^{\circ} \mathrm{C}$ for $45 \mathrm{~h}$, and $\mathbf{c}$ from the fragments of the PET-btl-1 in which the water was therein. VOCs detected: (1) acetic aldehyde; (2) acetone; (3) buta-

$(\mathrm{OH})$ and carboxyl $(\mathrm{COOH})$ end groups, species $\mathrm{Cn}$, terminated with EG units at both ends, and oligomers Dn, having $\mathrm{COOH}$ end groups at both ends, were identified.

The cyclic oligomers are predominant in the mass range between 550 and $2400 \mathrm{~m} / \mathrm{z}$, whereas in the mass range higher than $2400 \mathrm{~m} / \mathrm{z}$, the linear oligomers series (Bn, Cn and Dn) prevail (Fig. 2, enlargement of the mass spectrum from 2520 up to $2710 \mathrm{~m} / \mathrm{z}$ ). The mass spectrum also reveals the presence of linear PET oligomers with two (species B'n, C'n and D'n) or three (species B' n and C'n) DEG units along the chains. A molar amount of about $2.5 \%$ mol of DEG units in the PET-bottle1 was determined by ${ }^{1} \mathrm{H}-\mathrm{NMR}$ analysis (Fig. S4, protons DE1 and DE2). The ${ }^{1} \mathrm{H}-\mathrm{NMR}$ spectrum also reveals the presence of isophthalic acid units (about $2.5 \% \mathrm{~mol}$, protons $\mathrm{c}, \mathrm{d}, \mathrm{e})$, confirming the results from MALDI-TOF MS. A similar molar composition was found for all PET bottles studied.

The migration of cyclic and linear low molar mass PET oligomers (dimers up to tetramers) from PET bottle in a fat food simulant (ethanol 95\%) was observed by Ubeda nal; (4) 3-methyl butanal; (5) 1,3-dioxolane; (6) pentanal; (9) hexanal; (12) octanal; (13) 5-hepten-2-one, 6-methyl; (14) nonanal; (15) decanal; (16) 1-octanol; (17) undecanal; (EG) ethylene glycol; (18) 1-nonanol; and (19) dodecanal

et al. [46], whereas these oligomers were not found in aqueous food simulants (ethanol $10 \%$ or acetic acid $3 \%$ ). Some authors reported the presence of cycle PET trimers in PETbottled mineral waters $[31,56]$. Analysing the enlarged section from 600 up to $1100 \mathrm{~m} / \mathrm{z}$ of Fig. 2, we observed a series of homologous peaks, at $\mathrm{m} / \mathrm{z} 641.4,669.4,685.4$, $695.4,885.9,913.9,927.9,941.9,956,969.4,997.6$, and 1011.8 (labeled with the symbol *), which do not belong to the PET macromolecular chains. Based on their masses, we have assigned these unexpected peaks to the sodiated ions of epoxidized, partial epoxidized, and non-epoxidized triglycerides and diglycerides molecules. We hypothesize that these compounds may derive from an epoxidized soybean oil (ESBO) additive [6, 41, 47-49] probably used during the manufacturing of the PET bottles. Indeed, ESBO mainly consists of some triglycerides of the saturated palmitic (C16) and stearic (C18) fatty acids and of the epoxidized unsaturated ones, such as epoxidized oleic (C18:1E), diepoxidized linoleic (C18:2E), and triepoxidized linolenic (C18:3E) compounds. Taking into account the ESBO composition, 
Table 1 Volatile compounds detected by the HS-GC/MS analysis in six commercial Italian PET bottles

\begin{tabular}{lllllllll}
\hline Peak N & Retention time & Compounds & \multicolumn{2}{l}{ Normalized intensity ${ }^{\text {(a) }}$} & & \\
\cline { 4 - 8 } & & & Btl-1 & Btl-2 & Btl-3 & Btl-4 & Btl-5 & Btl-6 \\
\hline $\mathbf{1}$ & 1.23 & Acetic aldehyde & 31.0 & 100.0 & 95.4 & 49.0 & 78.5 & 74.5 \\
$\mathbf{2}$ & 1.45 & Acetone & 46.5 & 50.0 & 100.0 & 58.0 & 100.0 & 100.0 \\
$\mathbf{3}$ & 1.62 & Butanal & 2.0 & 2.5 & N.D & 8.0 & N.D & N.D \\
$\mathbf{4}$ & 1.86 & 3-Methyl butanal & 2.4 & 3.3 & 5.8 & N.D & 7.55 & 3.7 \\
$\mathbf{5}$ & 2.18 & 1.3-Dioxolane & 6.4 & 22.8 & 39.5 & 18.9 & 39.3 & 49.6 \\
$\mathbf{6}$ & 2.28 & Pentanal & 1.4 & 2.9 & 5.6 & 22.1 & 4.4 & 7.7 \\
$\mathbf{7}$ & 2.86 & Decane & N.D & 1.4 & 11.3 & N.D & N.D & N.D \\
$\mathbf{8}$ & 3.16 & Toluene & N.D & N.D & N.D & 4.0 & N.D & N.D \\
$\mathbf{9}$ & 4.10 & Hexanal & 5.2 & 4.26 & 22.6 & 8.2 & 49.0 & 6.9 \\
$\mathbf{1 0}$ & 6.60 & Heptanal & 4.6 & 3.7 & 15.5 & 6.9 & 7.3 & 5.4 \\
$\mathbf{1 1}$ & 7.83 & Dodecane & N.D & 1.8 & 23.9 & 11.1 & N.D & 32.0 \\
$\mathbf{1 2}$ & 11.05 & Octanal & 8.85 & 5.7 & 21.1 & 16.2 & 9.5 & 9.3 \\
$\mathbf{1 3}$ & 13.30 & 5-Hepten-2-one, 6-methyl & 13.6 & 25.3 & 39.0 & 45.6 & 49.8 & 51.0 \\
$\mathbf{1 4}$ & 14.95 & Nonanal & 23.8 & 11.8 & 87.3 & 26.3 & 55.2 & 19.5 \\
$\mathbf{1 5}$ & 17.96 & Decanal & 100.0 & 49.6 & 97.7 & 100.0 & 45.5 & 62.5 \\
$\mathbf{1 6}$ & 19.70 & 1-Octanol & 4.0 & 3.0 & 10.3 & 6.6 & 3.6 & 5.8 \\
$\mathbf{1 7}$ & 20.55 & Undecanal & 5.3 & 3.3 & 7.0 & 7.6 & 3.3 & 6.0 \\
$\mathbf{1 8}$ & 22.36 & 1-Nonanol & 2.1 & 2.9 & 4.9 & 10.3 & 2.0 & 4.9 \\
$\mathbf{1 9}$ & 23.60 & Dodecanal & 2.0 & 1.7 & 2.9 & 3.7 & 1.5 & 2.5 \\
\hline
\end{tabular}

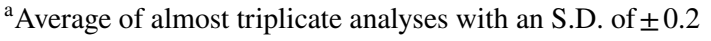

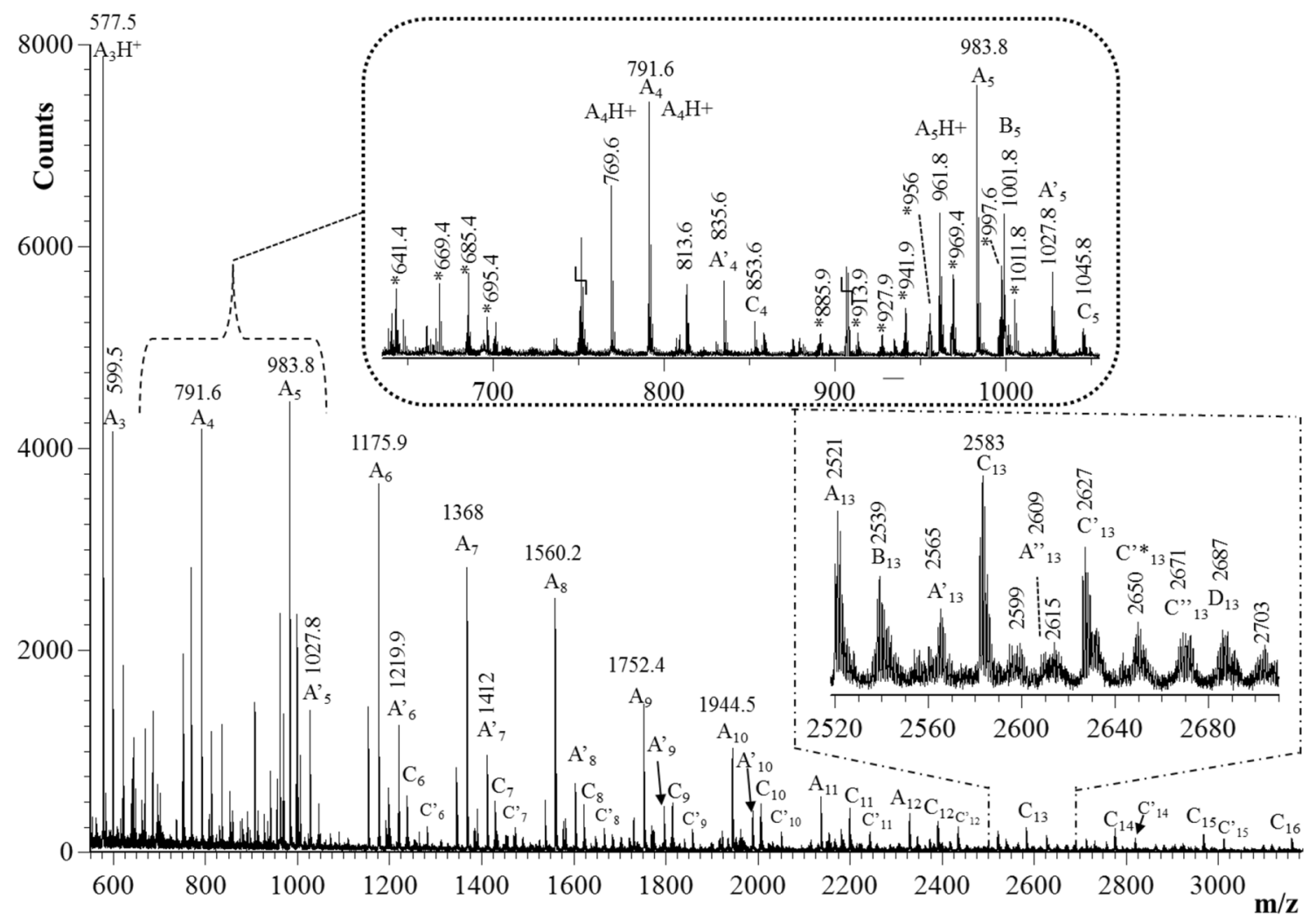

Fig. 2 MALDI-TOF mass spectrum of the TCE soluble fraction extracted from the PET-btl-1, recorded in reflectron mode, using DCTB as matrix 


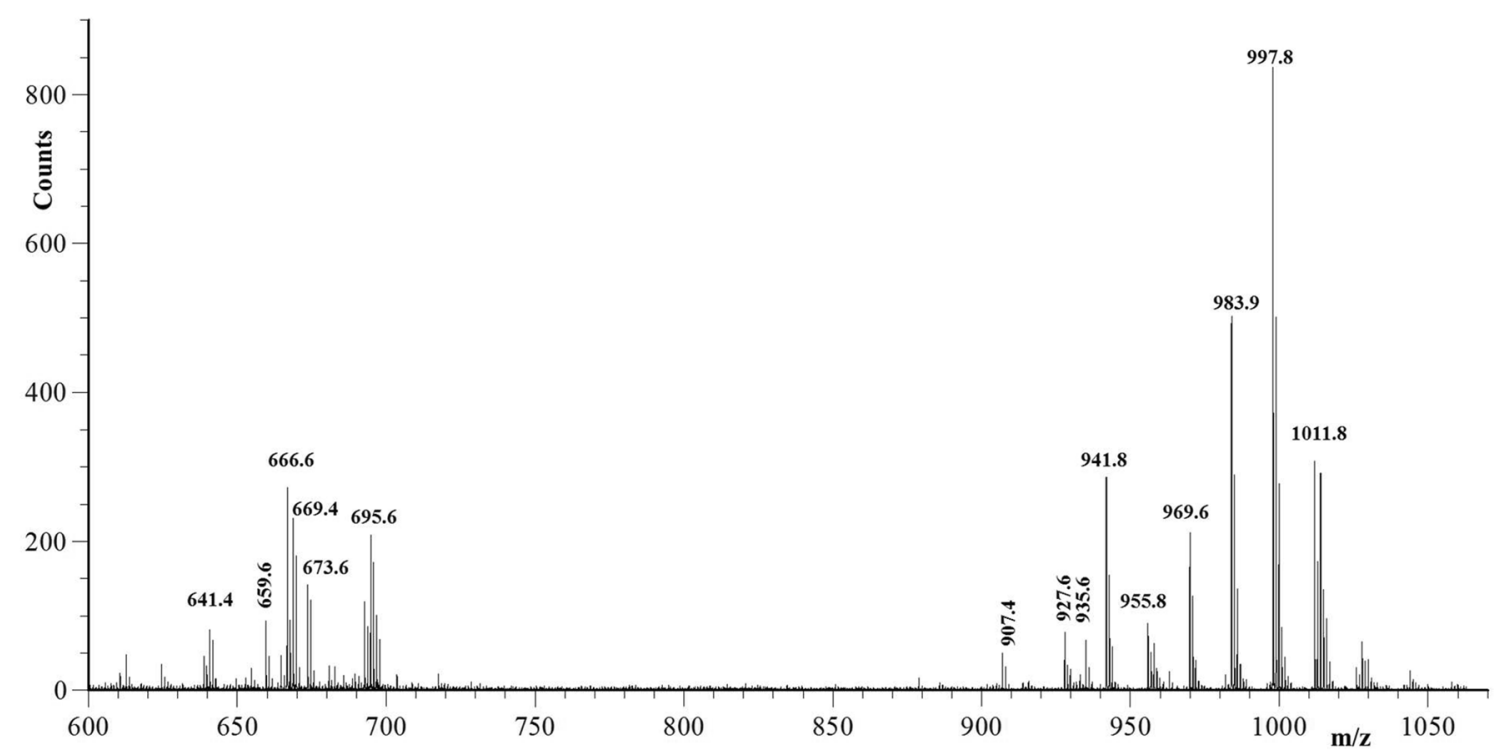

Fig. 3 MALDI-TOF mass spectrum of the virgin ESBO, recorded in reflectron mode, using DCTB as matrix

Fig. 4 Gas chromatogram of the neat ESBO analyzed by HS-GC/MS. VOCs detected: (3) butanal; $(\alpha)$ 2,4 methyl hexane; (6) pentanal; (9) hexanal; $(\beta)$ 3-heptanone; $(\eta)$ 2-penten-1ol; (11) dodecane; (12) octanal; (13) 5-hepten-2-one, 6-methyl; ( $\chi$ ) 2-heptenal; (14) nonanal; (15) decanal, (16) 1-octanol, and (18) 1-nonanol

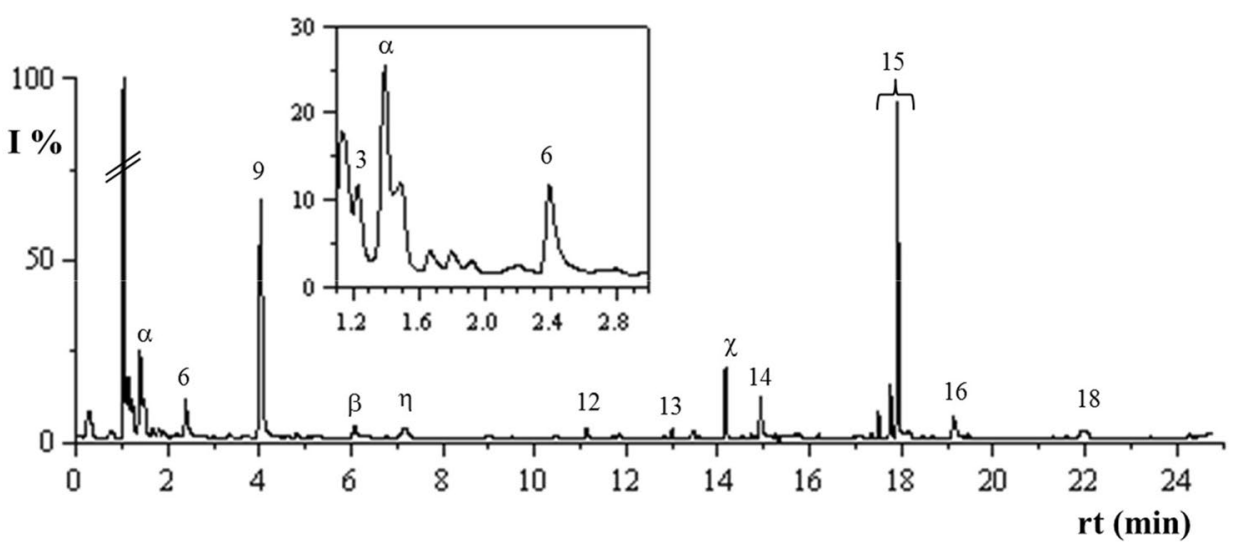

the intense mass peak at $m / z 997.8$ was assigned to the sodiated ions of the total epoxidized triglycerides of the linoleic acid (C18:2E), whereas the one at $\mathrm{m} / \mathrm{z} 1011.8$ belongs to the sodiated ions of the epoxidized triglycerides composed of two diepoxidized linoleic and one triepoxidized linolenic units (see Table S2). At the best of our knowledge, no literature data reported the use of ESBO as lubricant in the PET-bottle preparation.

To verify our findings, we developed btlg-PET blends containing fixed concentrations of the two additives ESBO and erucamide ((Z)-Docos-13-enamide), both authorized for the manufacture of food contact packaging due to their presence in the positive list of regulation n. 10/2011 [18].

\section{Development and analysis of Btlg-PET/ESBO blends}

Before the development of Btlg-PET/ESBO blends, we analyzed a commercial ESBO sample by both the MALDI-TOF
MS and the HS-GC/MS methods. The MALDI mass spectrum of a neat ESBO sample (Fig. 3) reveals a series of peaks related to the epoxidized di- and triglycerides (most abundant peak series), very similar to those observed in the extracted PET bottles samples discussed above (Fig. 2, Table S2). Figure 4 shows the HS-GC/MS chromatogram of the volatile organic compounds found in the neat ESBO sample. It highlights the presence of aliphatic aldehydes, such as butanal, pentanal, hexanal, octanal, nonanal, and decanal, as those observed in the HS-GC/MS analysis of all PET-bottle fragments analyzed (Figs. 1c and S3, Table 1). Likely, the presence of unsaturated aliphatic aldehyde (2-heptenal), ketones (6 methyl-5-hepten-2-one and 3-heptanone), aliphatic hydrocarbon (iso pentane), and aliphatic alcohols (pentanol, 2-ethyl-1-hexanol) was also detected.

The comparison of the HS-GC/MS chromatograms in Figs. 1c, 4 and S3 highlights that most of the VOCs (pentanal, hexanal, octanal, nonanal, decanal, 6 
Fig. 5 Gas chromatograms of HS-GC/MS analyses of the btlg-PET pellets melt-mixed a without and b with ESBO $1 \%$ w at $280{ }^{\circ} \mathrm{C}$ for $10 \mathrm{~min}$. VOCs detected: (1) acetic aldehyde; (2) acetone; (3) butanal; (4) 3-methyl butanal; (5) 1,3-dioxolane; (9) hexanal; $(\eta)$ 2-penten1ol; ( $\delta$ ) 2,4-nonadienal; (12) octanal; (13) 5-hepten-2-one, 6-methyl; $(\Delta)$ 2-octen-1ol; $(\varepsilon)$ 1(2-methoxyethoxy)2methyl2-propanol; $(\phi) 2$ nonen-1-ol (15) decanal; and (16) 1-octanol

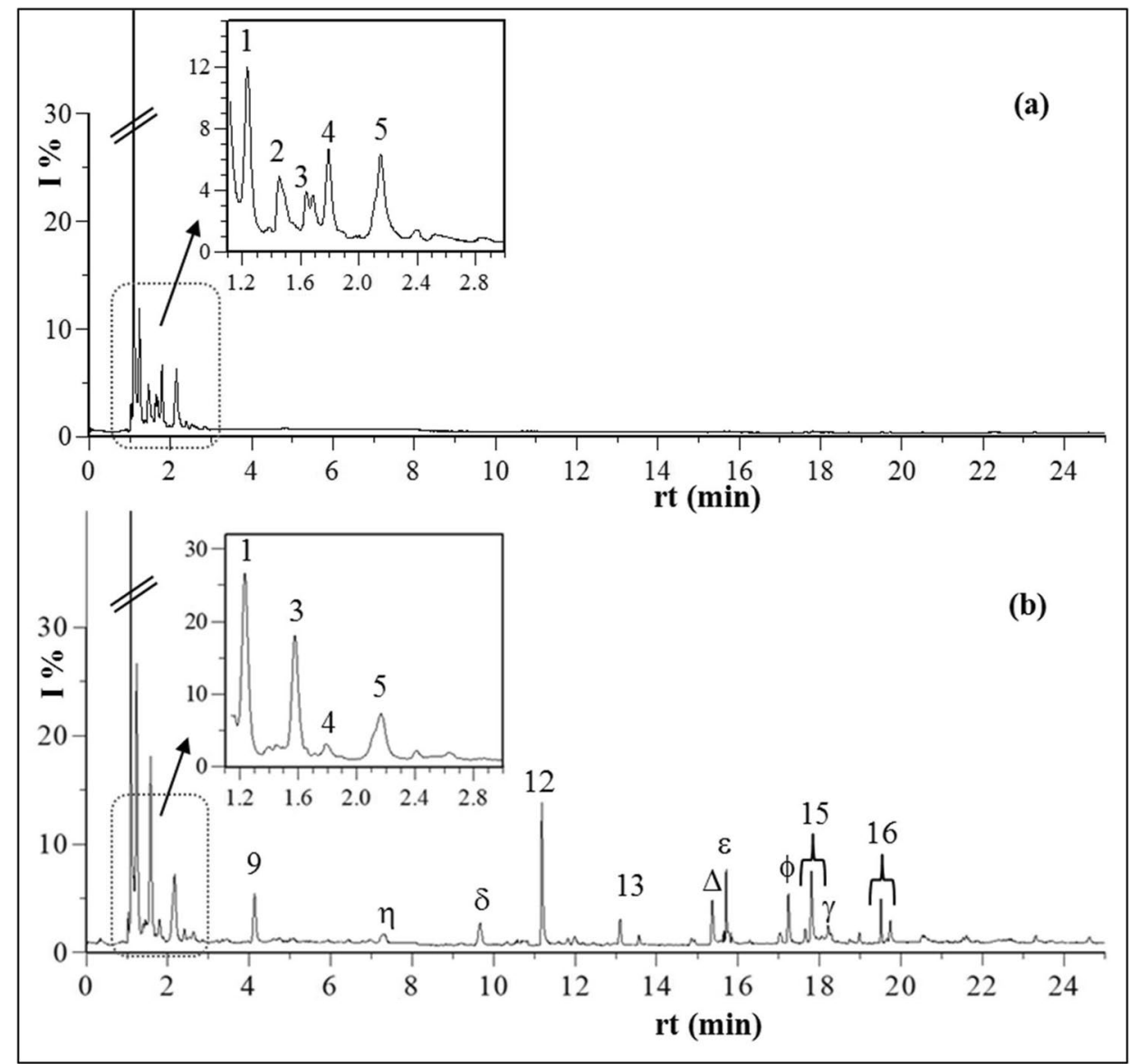

methyl-5-hepten-2-one and 3-heptanone, and aliphatic hydrocarbons) observed in all PET bottles (Table 1) could derive from an ESBO additive probably used for the preparation of the bottles, during the blow-moulding processes of the pre-forms.

These results suggest that the formation of the above VOCs (aliphatic aldehyde, ketone, and aliphatic alcohols) may also occur during the epoxidation process of soybean oil and not only when it is used during the manufacturing of the PET bottles.

To investigate the influence of this additive during the PET processing, we melt-mixed the virgin btlg-PET pellets with and without $1 \% \mathrm{w}$ of ESBO at $280{ }^{\circ} \mathrm{C}$ for 5,10 , and $20 \mathrm{~min}$. The gas chromatograms of the melt-mixed samples for 10 min of btlg-PET pellets without and with ESBO (1\%w) are showed in Fig. 5. The supposed formation of AA and 1,3-dioxolane is confirmed in the chromatogram of the melted neat btlg-PET pellets (Fig. 5a). In the melt-blended btlg-PET sample loaded with ESBO (Fig. 5b), besides the above volatile compounds, like those depicted in Fig. S3, was also observed the presence of saturated aldehydes (peaks $9,12,14,15$ ), ketone (peak 13), unsaturated aldehyde (peak $\delta$ ), and alcohols (peaks $\varepsilon, \phi, 16$ ), probably derived from this additive (Fig. 4) and its degradation products. Indeed, the presence of the species $\eta$ (2-penten-1ol), $\delta$ (2,4-nonadienal), $\Delta$ (2-octen-1ol); $\varepsilon$ (1(2-methoxyethoxy)2methyl2-propanol), and $\phi$ (2 nonen-1-ol), that may derive from the thermal degradation of the ESBO at $280{ }^{\circ} \mathrm{C}$, was also detected.

These data, together with those from MALDI-TOF MS analysis, confirm the use of ESBO as additive in the manufacturing of the PET bottles studied. In particular, the absence of the ESBO thermal degradation products as $\eta, \delta, \Delta, \varepsilon$, and $\phi$ in both MALDI-TOF MS and HS-GC/MS analyses of the virgin btlg-PET pellets and in the PET-bottle fragments (Figs. 1c and S3) suggests that ESBO is probably used in the blow-moulding processes (stretching and blowing) for manufacturing PET bottles. These processes are carried out at a blow temperature of about $105{ }^{\circ} \mathrm{C}$ that is the ideal temperature for orientation of PET and not at the high temperatures used for PET polymerization and pre-forms' preparation [50].

\section{Development and analysis of Btlg-PET/erucamide blends}

The use of erucamide, a primary fatty amide, as additive in the plastic manufacturing industry provides good 
Fig. 6 Gas chromatogram of HS-GC/MS analysis of the btlg-PET/erucamide (1\%w) melt-blended for $10 \mathrm{~min}$ at 280C. VOCs detected: (1) AA and (2) acetone

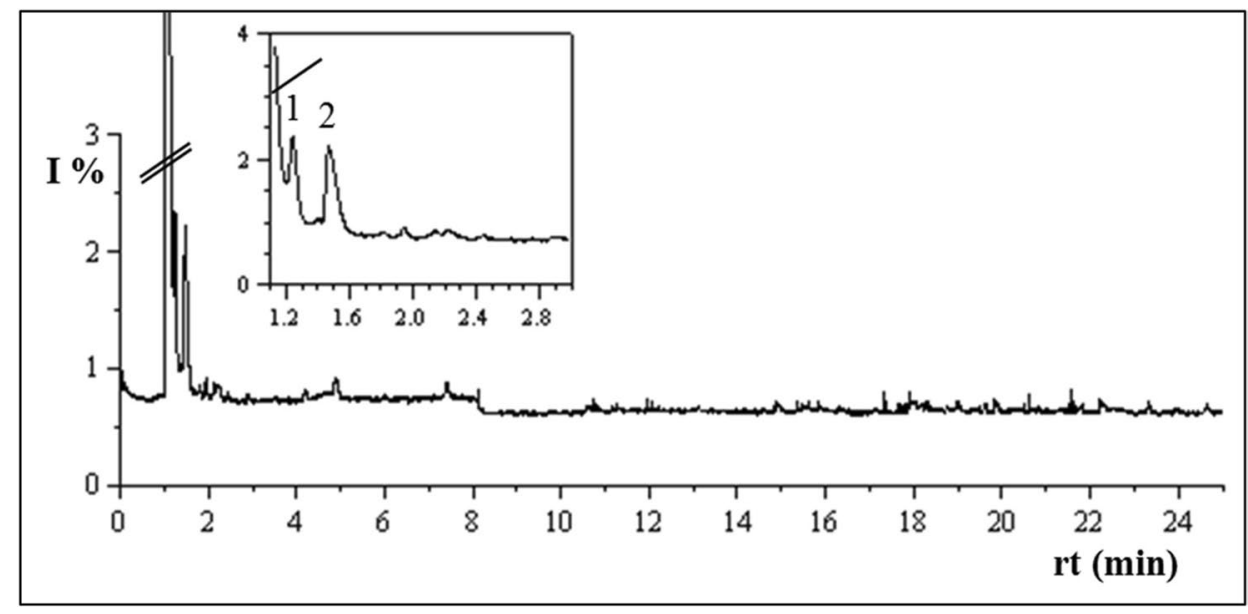

anti-blocking and slip properties, as well as overprinting and wet printing properties, and improved resistance to scratches and abrasion. Since it is generally used as lubricants during the manufacturing of the PET bottles [21, 48], to verify whether some VOCs observed in the PET bottles could derive from its use, we melt-blended the btlg-PET pellets with this compound $(1 \% \mathrm{w})$. The HS-GC/MS analyses of all btlg-PET/erucamide melt-blended samples showed two peaks relative to AA (peak 1) and acetone (peak 2), as highlighted in Fig. 6. These results give further evidence that the source of the VOCs identified by HS-GC/MS analysis in the PET bottles (Table 1) may derive from an ESBO additive used as lubricant during the manufacturing of the bottles.

\section{Studies on potential functional aldehydes scavangers}

AA scavengers are used in the PET bottles for mineral water to minimize AA content of the melt-processed polymeric materials, avoiding its migration into beverages, [7, 51-53]. Generally, to reduce the generation of AA during the PET pre-form production is used the 2-aminobenzamide, also known as anthranilamide [7, 51-53], for its low cost, efficiency, and ease of incorporation into PET material. It reacts with AA during the PET pre-form manufacturing as proposed by Mrozinski et al. [51, 52] (Scheme S1). In accord to the European Plastics Regulation No. 10/2011 (EU 2011), this additive can be used in PET bottles for water and beverages [18].

Moreover, the poly(m-xylene adipamide) MXD6, an efficient oxygen scavenger additive, which acts as passive polymer barrier reducing the oxygen permeation in the PET bottles used for the packaging of beverages and oils [54, 55], could be used as AA. However, some authors [21, 56] reported that the MXD6 layer can generate non-intentionally added substances (NIAS) in the PET bottles. Previously, MXD6 has been deeply characterized by MALDI-TOF MS and $\left({ }^{1} \mathrm{H}\right.$ and $\left.{ }^{13} \mathrm{C}\right)$-NMR [57]. The MALDI-TOF analysis revealed that the MXD6 chains are essentially terminated with carboxyl and amino moieties. These functional end groups could promote the exchange reactions with ester groups of PET leading to the formation of copolyesteramides [57]. Furthermore, the amino $\left(\mathrm{NH}_{2}\right)$ end groups of MXD6 can react with the aldehydes formed during the manufacturing of the PET bottles, acting as aldehyde scavenger. Bandi et al. [58] proposed that the coloration observed in a PET/ MXD6 (1:1 w/w) melt-mixed blend is due to conjugated chromophores generated by the condensation reactions of acetaldehyde and polyamide end groups.

To verify the efficiency of the anthranilamide and MXD6 as AA scavengers, we melt-processed the virgin btlg-PET pellets with $1 \% \mathrm{w}$ of each of them at $280{ }^{\circ} \mathrm{C}$ for different mixing times and analysed all samples by the HS-GC/MS. The VOCs detected in the melt-mixed Btlg-PET blends loaded with anthranilamide, (MXD6), anthranilamide/MXD6, and anthranilamide/MXD6/ESBO mixtures are reported in Table 2, whereas their corresponding gas chromatograms are showed in Figs. S5-S8.

Results on btlg-PET/anthranilamide blends highlighted the presence of AA only in the sample melt-mixed for 20 min, confirming the efficiency of anthranilamide as AA scavenger at lower mixing times (5-10 $\mathrm{min})$. On the contrary, the presence of acetone, 3-methyl butanal, 1,3-dioxolane, and 1,3-dioxolane was observed in all btlg-PET/ anthranilamide samples (Fig. S5). We assume that $1 \%$ w of anthranilamide is not sufficient to block all AA deriving from the degradation processes occurring during the longtime PET processing. Furthermore, it was not observed a clear increase of other compounds (butanal and 1,3-dioxolane) as melt-mixing time increases.

The btlg-PET/MXD6 blends show the absence of AA only in the samples at the lowest mixing time, suggesting that the AA formed at $5 \mathrm{~min}$ of processing has reacted with all the amino end groups of the MXD6 chains (Scheme S2). 
Table 2 Volatile compounds detected by HS-GC/MS analysis in melt-mixed Btlg-PET blends loaded with anthranilamide, poly(m-xylene adipamide) (MXD6), anthranilamide/MXD6, and anthranilamide/MXD6/epoxidized soybean oil (ESBO) mixtures

\begin{tabular}{|c|c|c|c|c|c|c|c|c|c|c|c|c|c|c|}
\hline \multirow[t]{4}{*}{ Species $^{\mathrm{a}}$} & \multirow[t]{4}{*}{ Retention time } & \multirow[t]{4}{*}{ Compounds } & \multicolumn{12}{|c|}{ Btlg- PET blends } \\
\hline & & & \multirow{2}{*}{\multicolumn{3}{|c|}{$\begin{array}{l}\text { Anthranilamide } \\
\begin{array}{l}\text { Melt-mixing time } \\
(\min )\end{array}\end{array}$}} & \multicolumn{3}{|c|}{ MXD6 } & \multicolumn{3}{|c|}{$\begin{array}{l}\text { Anthranilamide/ } \\
\text { MXD6 }\end{array}$} & \multicolumn{3}{|c|}{$\begin{array}{l}\text { Anthranilamide/ } \\
\text { MXD6/ESBO }\end{array}$} \\
\hline & & & & & & \multicolumn{3}{|c|}{$\begin{array}{l}\text { Melt-mixing time } \\
(\mathrm{min})\end{array}$} & \multicolumn{3}{|c|}{$\begin{array}{l}\text { Melt-mixing time } \\
(\mathrm{min})\end{array}$} & \multicolumn{3}{|c|}{$\begin{array}{l}\text { Melt-mixing } \\
\text { time (min) }\end{array}$} \\
\hline & & & 5 & 10 & 20 & 5 & 10 & 20 & 5 & 10 & 20 & 5 & 10 & 20 \\
\hline 1 & 1.23 & Acetic aldehyde & - & - & + & - & + & + & - & - & + & - & - & + \\
\hline 2 & 1.45 & Acetone & + & + & + & + & + & + & + & + & + & + & - & - \\
\hline 3 & 1.62 & Butanal & - & - & - & + & + & + & - & - & - & + & + & + \\
\hline 4 & 1.86 & 3-Methyl butanal & + & + & + & + & + & + & + & + & + & + & + & + \\
\hline 5 & 2.18 & 1,3-Dioxolane & + & + & + & + & + & + & + & + & + & + & + & + \\
\hline 6 & 2.28 & Pentanal & + & + & + & + & + & + & + & + & + & + & + & + \\
\hline 7 & 2.86 & Decane & - & - & - & - & - & - & - & - & - & - & + & + \\
\hline 9 & 4.10 & Hexanal & - & - & - & - & - & - & - & - & - & + & + & + \\
\hline$\eta$ & 7.30 & 2-Penten-1ol & - & - & - & - & - & - & - & - & - & + & + & + \\
\hline$\delta$ & 9.70 & 2,4-Nonadienal & - & - & - & - & - & - & - & - & - & + & + & + \\
\hline 12 & 11.05 & Octanal & - & - & - & - & - & - & - & - & - & + & + & + \\
\hline 13 & 13.30 & 5-Hepten-2-one, 6-methyl & - & - & - & - & - & - & - & - & - & - & + & + \\
\hline$\Delta$ & 15.30 & 2-Octen-1ol & - & - & - & - & - & - & - & - & - & + & + & + \\
\hline$\varepsilon$ & 15.70 & $\begin{array}{l}\text { 1(2-methoxyethoxy) } 2 \text { methyl } \\
\text { 2-propanol }\end{array}$ & - & - & - & - & - & - & - & - & - & + & + & + \\
\hline$\phi$ & 17.20 & 2-Nonen-1ol & - & - & - & - & - & - & - & - & - & + & + & + \\
\hline 15 & 17.97 & Decanal & - & - & - & - & - & - & - & - & - & + & + & + \\
\hline$\gamma$ & 18.20 & Pentenal, 2-ethyl-4- methyl & - & - & - & - & - & - & - & - & - & + & + & + \\
\hline 16 & 19.70 & 1-Octanol & - & - & - & - & - & - & - & - & - & + & + & + \\
\hline 17 & 20.60 & Undecanal & - & - & - & - & - & - & - & - & - & - & + & + \\
\hline EG & 22.00 & Ethylene glycol & - & - & - & - & - & - & - & - & - & + & + & + \\
\hline
\end{tabular}

+ detected; - not detected

${ }^{\text {a }}$ Species numbers correspond to those of Table 1

Acetone (peak 2) and traces of 3-methyl butanal (peak 4), 1,3-dioxolane (peak 5), and pentanal (peak 6) were also observed, independently of the melt-mixing time. The MALDI-TOF MS spectra of the btlg-PET/MXD6 samples, besides the expected cyclic and liner PET oligomers, revealed the formation of PET-MXD6 copolymers (species $X_{n, m}, Y_{n, m}$, and $Z_{n, m}$, in Table S3 and in Fig. 7). MXD6 oligomers, produced by the reaction of its amino end groups with AA (Scheme S2) such as the species Sn at m/z 1052.2 and 1298 (Table S3), were also observed. Data from both HS-GC/MS and MALDI-TOF MS analyses suggest that low amount $(1 \% \mathrm{w})$ of MXD6 could be useful as AA scavenger during the processing of the btlg-PET at mixing time lower than $10 \mathrm{~min}$ at $280^{\circ} \mathrm{C}$. Therefore, MXD6 can be used either as aldehyde scavenger and as active oxygen barrier additive $[54,55]$. Moreover, comparing the gas chromatograms from both btlg-PET/anthranilamide and btlg-PET/MXD6 blends, it was not observed any different contaminants derived from the MXD6 additive. However, further studies need to verify if high amounts $(>1 \% \mathrm{w})$ of the MXD6 could generate other contaminants.

We also developed and analyzed btlg-PET/MXD6-anthranilamide blends to verify whether the action of both scavengers could be antagonist, synergic, or independent. Their chromatograms (Fig. S7, Table 2) show the presence of AA only at 20 min of blending, confirming the efficiency and the synergic action of both compounds (anthranilamide and MXD6) as AA scavengers.

To corroborate the use of MXD6/anthranilamide mixture as efficient AA scavenger, we melt-mixed fragments of the PET-btl-1 with and without the MXD6-anthranilamide mixture and analysed the obtained samples (Fig. 8). The AA produced during the melt processing of the neat bottle fragments disappears in the blends melt-mixed for $5 \mathrm{~min}$ in the presence of the MXD6/anthranilamide mixture (inset in Fig. 8). However, the relative amount of the other species (i.e., peaks $12, \Delta, 15,16$, and $\varepsilon$ ) detected at high retention times does not change in the presence of these additives. 


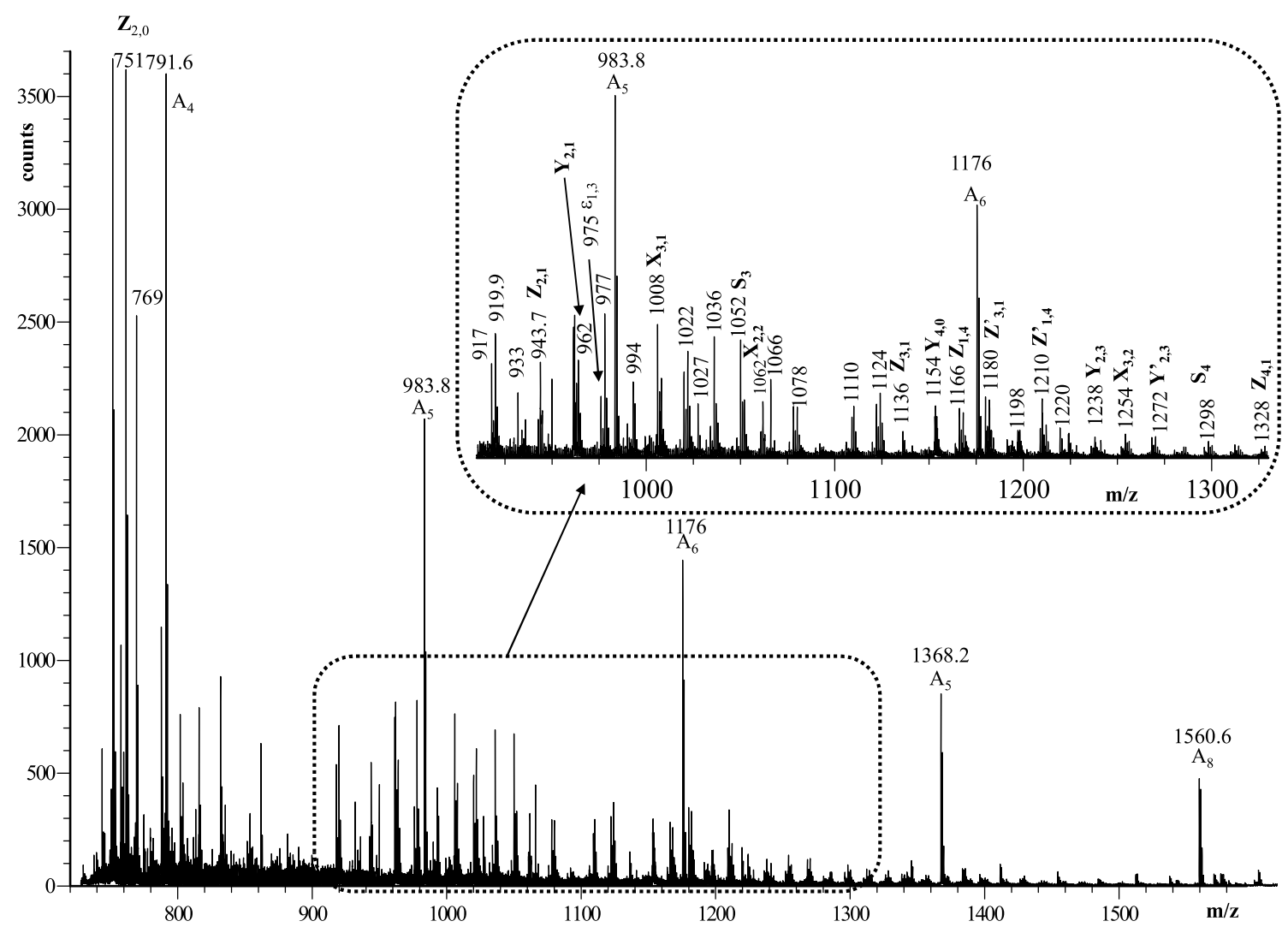

Fig. 7 MALDI-TOF mass spectrum of the TCE soluble fraction extracted from the btlg-PET melt-mixed with $1 \% \mathrm{w}$ of MXD6 at $280{ }^{\circ} \mathrm{C}$ for $5 \mathrm{~min}$

Very similar results were obtained by HS-GC/MS analysis of all PET-bottle fragments melt-mixed with MXD6/anthranilamide mixture for 10 and $20 \mathrm{~min}$.

Additionally, the efficiency of the MXD6/anthranilamide mixture as AA scavenger was tested by melt-mixing the btlg-PET with this mixture in the presence of the ESBO as lubricant, all additives at $1 \% \mathrm{w}$ (Table 2). The gas chromatograms of the obtained melted blends at 5, 10, and $20 \mathrm{~min}$ are displayed in Fig. S8. The AA is detected only at meltmixing time of 20 min, thus confirming the ability of both compounds (MXD6 and anthranilamide) as AA scavenger. Nevertheless, the presence of short- and long-chain aldehydes (butanal, pentanal, hexanal, octanal, nonanal, and decanal), ketone (compound 13), and alcohols (compounds, $\eta, \Delta, \varepsilon, \phi, \gamma$ and 16) was also depicted, as observed in the melt-blended btlg-PET/ESBO samples (Fig. 4).

These findings corroborate our hypothesis that these VOCs, similar to those observed in the fragments of the PET bottles investigated (Figs. 1c and S3), are due to an ESBO lubricant used during the manufacturing process. The relative intensity of the volatile degradation products, $\eta, \varepsilon, \phi$, and $\gamma$ increases as raises the amount of the additive ESBO. Since these volatile compounds were observed only in samples containing ESBO melt-mixed at $280^{\circ} \mathrm{C}$ (Table 2, Figs. 5, S8 and S9), we suppose that the ESBO additive detected in PET bottles analysed was not used in the polymerization process of the btlg-PET, which is carried out at $280{ }^{\circ} \mathrm{C}$, but probably during the blow-moulding processes of the PET pre-forms.

\section{Conclusions}

A simple analytical method based on HS-GC/MS technique allowed the detection of several VOCs in six Italian mineral waters packaged in PET bottles. The same VOCs were detected in all primary packaging (PET bottles). The MALDI-TOF MS analysis of all PET-bottle fragments of the six Italian waters selected highlighted the presence of ESBO as additive. The volatile sub-products present in an ESBO sample were identified by HS-GC/MS. Most of them correspond to those observed in the PET-bottle fragments. The comparison of HS-GC/MS and MALDI-TOF MS analyses of virgin Btlg-PET pellets, PET fragments from the PET bottles and neat ESBO additive demonstrated that some VOCs come from an ESBO additive likely loaded during the blow-moulding processes for PET bottles preparation. 
Fig. 8 Gas chromatograms of HS-GC/MS analysis of the PET-btl-1 melt-mixed at $280{ }^{\circ} \mathrm{C}$ for 5 min a without and with $\mathbf{b}$ MXD6 (1\%w) and anthranilamide $(1 \% \mathrm{w})$. VOCs detected: (1) acetic aldehyde; (3) butanal; (4) 3-methyl butanal; (5) 1,3-dioxolane; (6) pentanal; (12) octanal; $(\Delta)$ 2-octen-1ol; $(\varepsilon)$ 1(2-methoxyethoxy)2methyl2-propanol; $(\phi) 2$ nonen-1-ol (15) decanal; (16) 1-octanol and (EG) ethylene glycol

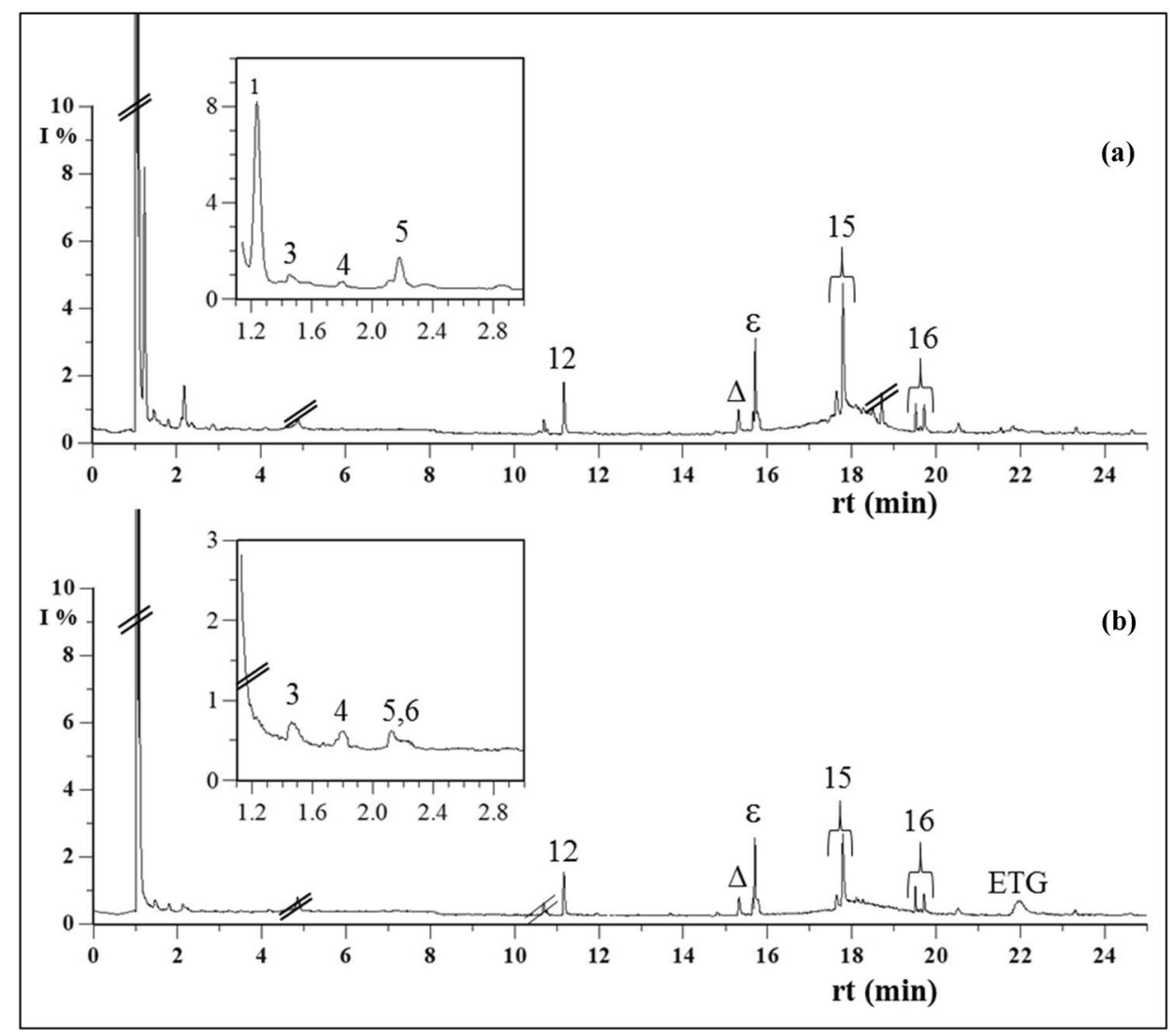

Additional studies carried out to find potential AA scavengers showed that MXD6, which is an efficient oxygen scavenger additive, can also be used as AA scavenger even in the presence of anthranilamide, a known AA scavenger. Our results give fundamental information about the origin of VOCs in the Italian PET bottles analysed and their corresponding packaged mineral waters.

Supplementary Information The online version contains supplementary material available at https://doi.org/10.1007/s00217-022-03973-5.

Acknowledgements Many thanks are due to Mrs. Gaetano Pastorelli and Roberto Rapisardi for the technical support.

Funding Financial support was provided by the Ministry of University and Scientific Research (MIUR) of Italy and the National Council of Research (CNR) of Italy. Many thanks are due to POR FSE Sicily 2020 e Project: "Polymeric systems: innovative aspects and applications in the biomedical and agri-food fields and SPIN OFF of Polymers", Call $11 / 2017$, "Strengthening employability in the R\&D system and the emergence of research spin offs in Sicily", that granted this work.

\section{Declarations}

Conflict of interest The authors declare no conflict of interest.

Compliance with ethics requirements This article does not contain any studies with human participants or animals performed by any of the authors.
Informed consent Not applicable.

Open Access This article is licensed under a Creative Commons Attribution 4.0 International License, which permits use, sharing, adaptation, distribution and reproduction in any medium or format, as long as you give appropriate credit to the original author(s) and the source, provide a link to the Creative Commons licence, and indicate if changes were made. The images or other third party material in this article are included in the article's Creative Commons licence, unless indicated otherwise in a credit line to the material. If material is not included in the article's Creative Commons licence and your intended use is not permitted by statutory regulation or exceeds the permitted use, you will need to obtain permission directly from the copyright holder. To view a copy of this licence, visit http://creativecommons.org/licenses/by/4.0/.

\section{References}

1. Rodwan Jr JG (2018) Significant but slower, growth for bottled water in 2018. Available online: https://bottledwater.org/wpcontent/uploads/2020/03/2018BottledWaterStats_pub2019.pdf. Accessed 10 Sep 2021

2. Welle F (2011) Twenty years of PET bottle to bottle recycling - an overview. Resour Conserv Recy 55(11):865-875. https://doi.org/ 10.1016/j.resconrec.2011.04.009.Accessed12Sept2021

3. Begley TH, Dennison JL, Hollifield HC (1990) Migration into food of polyethylene terephthalate (PET) cyclic oligomers from PET microwave susceptor packaging. Food Addit Contam 7:797-8034 
4. Ashby R (1988) Migration from polyethylene terephthalate under all conditions of use. Food Addit Contam 5:485-492

5. PETCore website (2017). https://www.petcore-europe.org/newse vents/110-pet-collection-recycling-rates-europe-significantly2016.html, written 18 December 2017. Accessed 31 May 2021

6. Coniglio MA, Fioriglio C, Laganà $P(2020)$ The bottled water. In: Non-intentionally added substances in PET-bottled mineral water. Springer International Publishing, pp 11-28, https://doi.org/10. 1007/978-3-030-39134-82.

7. Welle F (2015) The Facts about PET. Research. https://doi.org/ 10.13140/RG.2.1.3043.2726

8. Romao W, Franco MF, Corilo YE, Eberlin MN, Spinace MA, De Paoli M-A (2009) Poly (ethylene terephthalate) thermo-mechanical and thermo-oxidative degradation mechanisms. Polvm Deg Stab 94:1849-1859. https://doi.org/10.1016/j.polymdegradstab. 2009.05.017

9. Ciolacu CFL, Choudhury NR, Dutta NK (2006) Colour formation in poly(ethylene terephthalate) during melt processing. Polvm Deg Stab 91:875-885. https://doi.org/10.1016/j.polymdegradstab. 2005.06.021

10. Samperi F, Puglisi C, Alicata R, Montaudo G (2004) Thermal degradation of poly(ethylene terephthalate) at the processing temperature. Polvm Deg \& Stab 83:3-10. https://doi.org/10.1016/ S0141-3910(03)00166-6

11. Carroccio S, Rizzarelli P, Scaltro G, Puglisi C (2008) Comparative investigation of photo- and thermal-oxidation processes in poly(butylene terephthalate). Polymer 49:3371-3381. https://doi. org/10.1016/j.polymer.2008.05.015

12. Ciolacu FCL, Choudhury NR, Dutta N, Voelcker NH (2006) MALDI-TOF MS and DIOS-MS investigation of the degradation and discoloration of poly(ethylene terephthalate). Macromolecules 39:7872-7881. https://doi.org/10.1021/ma060757w

13. Weidner S, Kuhn G, Friedrich J, Schröder H (1996) Plasmaoxidative and chemical degradation of poly(ethylene terephthalate) studied by matrix-assisted laser desorption/ionization mass spectrometry. Rapid Commun Mass Spectrom 10:40-46

14. Weidner S, Kuehn G, Werthmann B, Schroeder H, Just U, Borowski R, Decker R, Schwarz B, Schmuecking I, Seifert I (1997) A new approach of characterizing the hydrolytic degradation of poly(ethylene terephthalate) by MALDI-MS. J Polym Sci A Polym Chem 35:2183-2192

15. Paci M, La Mantia FP (1998) Competition between degradation and chain extension during processing of reclaimed poly(ethylene terephthalate. Polvm Deg \& Stab 61:417-420

16. Villain F, Coudane J, Vert M (1995) Thermal degradation of polyethylene terephthalate: study of polymer stabilization. Polym Deg \& Stab 49:393-397

17. Lecomte HA, Liggat JJ (2006) Degradation mechanism of diethylene units in a terephthalate polymer. Polym Deg Stab 91:681-689. https://doi.org/10.1016/j.polymdegradstab.2005.05.028

18. Commission E (2011) Commission Regulation (EU) N 10/2011 of 14 January 2011 on plastic materials and articles intended to come into contact with food. Off J Eur Comm L12:1-89

19. Cincotta F, Verzera A, Tripodi G, Condurso C (2018) Non-intentional added substances in PET bottled mineral water during the shelf-life. Eur Food Res Technol 244:433-439. https://doi.org/10. 1007/s00217-017-2971-6

20. Choodum A, Thavarungkul P, Kanatharana P (2007) Acethaldehyde residue in polyethylene terephthalate (PET) bottles. J Environ Sci Health B 42:577-583. https://doi.org/10.1080/03601 230701391856

21. Bach C, Dauchy X, Chagnon M-C, Etienne S (2012) Chemical compounds and toxicological assessments of drinking water stored in polyethylene terephtalate (PET) bottles: a source of controversy reviewed. Water Res 46:571-583. https://doi.org/10. 1016/j.watres.2011.11.062
22. Li Bo, Wang Z-W, Qin-Bao C-Y (2016) Study of the migration of stabilizer and plasticizer from polyethylene terephthalate into food simulants. J Chromatogr Sci 54(6):939-951. https://doi.org/ 10.1093/chromsci/bmw025

23. Linssen J, Reitsma H, Cozijnsen J (1995) Static headspace gas chromatography of acetaldehyde in aqueous foods and polythene terephthalate. Z Lebensm Unters Forsch 201:253-255

24. Ceretti E, Zani C, Zerbini I, Guzzella L, Scaglia M, Berna V, Donato F, Monarca S, Feretti D (2010) Comparative assessment of genotoxicity of mineral water packed in polyethylene terephthalate (PET) and glass bottles. Water Res 44:1462-1470. https://doi.org/ 10.1016/j.watres.2009.10.030

25. Nawrocki J, Dabrowsk A, Borcz A (2002) Investigation of carbonyl compounds in bottled waters from Poland. Water Res 36:4893-4901. https://doi.org/10.1016/S0043-1354(02)00201-4

26. Sugaya N, Nakagawa T, Sakurai K, Morita M, Onodera S (2001) Analysis of aldehydes in water by head space-GC/MS. J Health Sci 47:21-27. https://doi.org/10.1248/jhs.47.21

27. Strube A, Buettner A, Groetzinger C (2009) Characterization and identification of a plastic-like off-odor in mineral water. Water Sci Technol 9:299-309

28. Al Rayes L, Saliba CO, Ghanem A, Randon J (2012) BTES and aldehydes analysis in PET-bottled water in Lebanon. Food Addit Contam Part B 5:221-227. https://doi.org/10.1080/19393210. 2012.698311

29. Schmid P, Welle F (2020) Chemical migration from beverage packaging materials - a review. Beverages 6:37. https://doi.org/ 10.3390/beverages6020037

30. Bentayeb K, Batlle R, Romero J, Nerín C (2007) UPLC-MS as a powerful technique for screening the nonvolatile contaminants in recycled PET. Anal Bioanal Chem 388:1031-1038. https://doi. org/10.1007/s00216-007-1341-9

31. Mutsuga M, Tojima T, Kawamura Y, Tanamoto K (2005) Survey of formaldehyde, acetaldehyde and oligomers in polyethylene terephthalate food-packaging materials. Food Addt Contan 22:783-789. https://doi.org/10.1080/02652030500157593

32. Bach C, Dauchy X, Severin I, Munoz JF, Etienne S, Chagnon M-C (2013) Effect of temperature on the release of intentionally and non-intentionally added substances from polyethylene terephthalate (PET) bottles into water: chemical analysis and potential toxicity. Food Chem 139:672-680. https://doi.org/10.1016/j.foodc hem.2013.01.046

33. Bach C, Dauchy X, Severin I, Munoz J-F, Etienne S, Chagnon M-C (2014) Effect of sunlight exposure on the release of intentionally and/or non-intentionally added substances from polyethylene terephthalate (PET) bottles into water: chemical analysis and in vitro toxicity. Food Chem 162:63-71. https://doi.org/10. 1016/j.foodchem.2014.04.020

34. Bach C, Dauchy X, David L, Etienne S (2011) Physico-chemical study of PET bottles and PET bottled water. Matér Techn 99:391408. https://doi.org/10.1051/mattech/2011006

35. Ioannidou MD, Samouris G, Achilias DS (2016) Acetaldehyde contamination of water, alcoholic, and non-alcoholic beverages stored in glass or plastic bottles. Toxicol Environ Chem 98(10):1183-1190. https://doi.org/10.1080/02772248.2015.11155 05

36. Dabrowska A, Borcz A, Nawrocki J (2003) Aldehyde contamination of mineral water stored in PET bottles. Food Addit Contam 20:1170-1177. https://doi.org/10.1080/02652030310001620441

37. Cardozo IMM, Pereira dos Anjos J, Campos O, da Rocha F, de Andrade JB (2021) Exploratory analysis of the presence of 14 carbonyl compounds in bottled mineral water in polyethylene terephthalate (PET) containers. Food Chem 365:130475. https://doi. org/10.1016/j.foodchem.2021.130475

38. Abe Y, Kobayashi N, Yamaguchi M, Mutsuga M, Ozaki A, Kishi E, Sato K (2021) Determination of formaldehyde and 
acetaldehyde levels in poly(ethylene terephthalate) (PET) bottled mineral water using a simple and rapid analytical method. Food Chem 344:128708. https://doi.org/10.1016/j.foodchem.2020. 128708

39. Takeda K, Katoh S, Nakatani N, Sakugawa H (2006) Rapid and highly sensitive determination of low-molecular-weight carbonyl compounds in drinking water and natural water by preconcentration HPLC with 2, 4-dinitrophenylhydrazine. Anal Sci 22:1509-1514

40. Kim HJ, Shin HS (2011) Simple and automatic determination of aldehydes and acetone in water by headspace solid-phase microextraction and gas chromatography-mass spectrometry. J Sep Sci 34:693-699. https://doi.org/10.1002/jssc.201000679

41. Lau O, Wong WS (2000) Contamination in food from packaging material. J Chromatogr A 882:255-270

42. LoPachin RM, Gavin T (2014) Molecular mechanisms of aldehyde toxicity: a chemical perspective. Chem Res Toxicol 27:10811109. https://doi.org/10.1021/tx5001046

43. Thoden van Velzen EU, Brouwer MT, Stärker C, Welle F (2020) Effect of recycled content and rPET quality on the properties of PET bottles, part II: migration. Packag Technol Sci 33:359-371. https://doi.org/10.1002/pts.2528

44. Gehring C, Welle F (2018) Migration Testing of polyethylene terephthalate: comparison of regulated test conditions with migration into real food at the end of shelf life. Packag Technol Sci 31:771-780. https://doi.org/10.1002/pts.2291

45. Silva Freitas FL, Chinellato AC, Pereira Filho ER, Cruz SA (2020) Evaluation of the effect of additives on thermo-oxidative and hydrolytic stabilization of recycled post-consumer poly (ethylene terephthalate) using Design of Experiments. Polym Test 81:106275. https://doi.org/10.1016/j.polymertesting.2019.106275

46. Ubeda S, Aznar M, Nerín C (2018) Determination of oligomers in virgin and recycled polyethylene terephthalate (PET) samples by UPLC-MS-QTOF. Anal Bioanal Chem 410:2377-2384. https:// doi.org/10.1007/s00216-018-0902-4

47. Bueno-Ferrer C, Jiménez A, Garrigòs MC (2010) Migration analysis of epoxidized soybean oil and other plasticizers in commercial lids for food packaging by gas chromatography-mass spectrometry. Food Addit Contam 27:1469-1477. https://doi.org/ 10.1080/19440049.2010.502129

48. Hahladakisa JN, Velis CA, Weber R, Iacovidou E, Purnell P (2018) An overview of chemical additives presents in plastics: Migration, release, fate and environmental impact during their use, disposal and recycling. J Hazard Mater 344:179-199. https:// doi.org/10.1016/j.jhazmat.2017.10.014

49. Pedersen GA, Jensen LK, Fankhauser A, Biedermann S, Petersen JH, Fabech B (2008) Migration of epoxidized soybean oil (ESBO) and phthalates from twist closures into food and enforcement of the overall migration limit. Food Addit Contam 25:503-510. https://doi.org/10.1080/02652030701519088

50. Manufacturing of Plastic Bottles (PET) http://newengineeringp ractice.blogspot.com/2011/08/manufacturing-of-plastic-bottles. html. Accessed 30 Sep 2021

51. Mrozinski BA, Lofgren EA, Jabarin SA (2012) Acetaldehyde scavengers and their effects on thermal stability and physical properties of poly(ethylene terephthalate). J Appl Polym Sci 125:2010-2021. https://doi.org/10.1002/app.36287

52. Mrozinski BA, Kim YW, Lofgren EA, Jabarin SA (2013) Chemistry of the Interactions of Acetaldehyde Scavengers for Poly(ethylene terephthalate). J Appl Polym Sci 130:4191-4200. https://doi.org/10.1002/APP.39702

53. Franz R, Gmeiner M, Gruner A, Kemmer D, Welle F (2016) Diffusion behaviour of the acetaldehyde scavenger 2-aminobenzamide in polyethylene terephthalate for beverage bottles. Food Addit Contam Part A 33(2):364-372. https://doi.org/10.1080/ 19440049.2015.1128566

54. Muller K, Sangerlaub S, Kramer A, Huber C, Fritsch K (2011) Temperature-dependent oxygen permeation through PET/MXD6barrier blend bottles with and without oxygen absorber. Brew Sci 64(11-12):161-167

55. Saengerlaub S, Mueller K (2017) Long-time performance of bottles made of PET blended with various concentrations of Oxygen Scavenger Additive Stored at Different Temperature. Packag Technol Sci 30:45-58. https://doi.org/10.1002/pts.2283

56. Franz R, Welle F (2008) Investigation of non-intentionally added substances (NIAS) in PET bottles and closures. In: Poster Presentation at the 4th International Symposium on food packaging, 19-21 November 2008, Prague, Czech Republic. https://www.ivv. fraunhofer.de/content/dam/ivv/en/documents/Forschungsfelder/ Produktsicherheit-und-analytik/NIAS_in_PET_bottles_and_closu res.pdf. Accessed 15 oct 2021

57. Samperi F, Montaudo MS, Battiato S, Carbone D, Puglisi C (2010) Characterization of copolyesteramides from reactive blending of PET and MXD6 in the molten state. J Polym Sci Part A-Polym Chem 48:5135-5155. https://doi.org/10.1002/pola. 24312

58. Bandi S, Mehta S, Schiraldi DA (2005) The mechanism of color generation in poly(ethyleneterephthalate)/polyamide blends. Polym Deg Stab 88:341-348. https://doi.org/10.1016/j.polym degradstab.2004.11.009

Publisher's Note Springer Nature remains neutral with regard to jurisdictional claims in published maps and institutional affiliations. 